\title{
Equilibruim Approach of Asset Pricing under Lévy Process
}

\author{
Jun Fu, Hailiang Yang* \\ Department of Statistics and Actuarial Science, The University of Hong Kong, Pokfulam Road, Hong Kong
}

\begin{abstract}
This work considers the equilibrium approach of asset pricing for Lévy process. It derives the equity premium and pricing kernel analytically for the stock price process, obtains an equilibrium option pricing formula, and explains some empirical evidence such as the negative variance risk premium, implied volatility smirk, and negative skewness risk premium by comparing the physical and risk-neutral distributions of the log return. Different from most of the current studies in equilibrium pricing under jump diffusion models, this work models the underlying asset price as the exponential of a Lévy process and thus allows nearly an arbitrage distribution of the jump component.
\end{abstract}

Keywords: Pricing; Equilibrium approach; Lévy process; Equity risk premium; Variance risk premium

\section{Introduction}

As is known to all, it has been long observed that jumps exist in asset prices. And various stock price processes with jump components have been proposed in the past literature, such as pure jump process by Cox and Ross (1976), jump diffusion process by Merton (1976) and Kou and Wang (2004), and jump diffusion process with short noise by Altmann et al. (2008) and Morenno et al. (2011). A very general jump diffusion model, which has been commonly used, is Lévy process, since it allows the jump component to have infinite activity and admits nearly an arbitrary distribution. Some special cases of this kind of jump component include the inverse Gaussian model of Barndorff-Nielsen (1997), the generalized hyperbolic class of Eberlein et al. (1998), the variance-gamma (VG) model of Madan et al. (1998), the CGMY model of Carr et al. (2002), and the finite moment log-stable model of Carr and $\mathrm{Wu}$ (2003). The jump component can capture the rare-event risk and make the asset return non-Gaussian distributed, and the study of option pricing with this rare-event risk and risk measure for the non-Gaussian distributed returns can be referred to Martzoukos and Trigeorgis (2002) and Rossello (2008).

By using a jump diffusion model in the production economy of Cox et al. (1985), a pricing kernel can be obtained and it provides the analytical relation between the physical and risk-neutral distributions of the $\log$ return. And regarding this relation, there are some important phenomena which have been observed and studied. First, the risk-neutral variance is larger than physical one. This phenomenon is referred to as the negative variance risk premium in Coval and Shumway (2001), Bakshi and Kapadia (2003), and Carr and Wu (2009), since it means that the long position in a variance swap contract is expected to lose rather than to gain. Second, the risk-neutral skewness is negative as observed by Rubinstein (1994) and Ait-Sahalia and Andrew (1998). This asymmetric distribution can result in the downward slope of the implied volatility smirk. Lastly, the risk-neutral skewness is more negative than the physical skewness, or called negative skewness risk premium,

*Corresponding author. Email: hlyang@hku.hk 
as studied by Bakshi et al. (1997). Similar to the first phenomenon, it means that the return of a skewness contract is negative.

The purpose of this paper is to establish a general equilibrium model with both diffusion and jump risks, under which an analytical form of the pricing kernel can be obtained, and the above mentioned empirical evidence can be explained through the relation between the physical and risk-neutral measures implied from the pricing kernel. Many similar questions have also been studied in the past, such as in Pan (2002), Liu and Pan (2003), and Liu et al. (2005), but they all impose some strict assumptions on the distribution of the jump component, such as constant jump size, zero mean of jump size, or normally distributed jump size. Removing these assumptions, Zhang et al. (2010) allows an arbitrary distribution for the jump size, but just uses the increment of a Poisson process to model the jump intensity. Therefore, compared to these past works, our study under Lévy process is more general in the sense that it uses the Lévy kernel to model the jump component, allowing not only an arbitratry distribution but also infinite activity for the jump intensity. Furthermore, in Zhang et al. (2010), although the pricing kernel and equilibrium option pricing formula can be obtained without imposing any assumption on the distribution of the jump size, the explanation of the empirical evidence, which is the most important part of the article, only focuses on the simplest case where the jump size is a constant. In constrast to this, in this article, an arbitrary Lévy kernel is always used for the jump component not only in the theoretical results but also in the study of the empirical phenomena. Therefore, it generalizes the explanantion of the empirical evidence to a more advanced model.

In the production economy of Cox et al. (1985) with only a single physical good used for both consumption and investment, we model the single production process as an ordinary exponential of a Lévy process. Under this model, firstly, the equity premium and pricing kernel can be obtained from an investment and consumption optimization problem for a representative investor with constant-relative-risk-averse (CRRA) utility. Then, from this pricing kernel, the equilibrium option pricing formula is derived, and it can be shown to be equivalent to the Bakshi and Madan (2000)'s inverse Fourier transformation formula which is based on the risk-neutral distribution of the log return. Lastly, which is the most important part, we analytically compare the risk-neutral and physical distributions, and explain the empirical evidence mentioned above including the negative variance risk premium, implied volatility smirk, and negative skewness risk premium. Moreover, an empirical application to the S\&P 500 index is also provided. In summary, this paper analyzes the effect of the jump component on the equity risk premium and on the link between the physical and risk-neutral measures, and furthermore studies the cause of the empirical phenomena from the perspective of jump risk.

The following sections of this paper are organized as follows. Section 2 introduces the exponential Lévy model for the single production process. Section 3 deriveds the equity premium and pricing kernel. Based on these results, the equilibrium option pricing is presented in Section 4. And Section 5 explains the empirical evidence. Section 6 concludes this paper.

\section{The Price Model}

In the production economy of Cox et al. (1985) with only a single physical good used for both consumption and investment, there is only one production process. And we assume that the production process $\left\{S_{t}\right\}_{t \geq 0}$ transforms like an exponential of a Lévy process. Let $\left\{X_{t}\right\}_{t \geq 0}$ be this Lévy process on $\mathbb{R}, \nu_{X}$ its Lévy kernel, and $J_{X}$ its jump measure, which is a Poisson random measure on $[0, \infty) \times \mathbb{R}$ with intensity measure $\nu_{X}(d x) d t$, and denote a one-dimensional standard Brownian motion by $\left\{B_{t}\right\}_{t \geq 0}$. If $\nu_{X}$ is a Radon measure on $\mathbb{R} \backslash\{0\}$ and 
satisfies

$$
\int_{|x|<1}|x|^{2} \nu_{X}(d x)<\infty \quad \int_{|x| \geq 1} \nu_{X}(d x)<\infty,
$$

then $\left\{X_{t}\right\}_{t \geq 0}$ admits the following Lévy-Itô decomposition

$$
X_{t}=\mu t+\sigma B_{t}+X_{t}^{l}+\lim _{\varepsilon \rightarrow 0} \tilde{X}_{t}^{\varepsilon}
$$

where

$$
\begin{aligned}
X_{t}^{l} & =\int_{0}^{t} \int_{|x| \geq 1} x J_{X}(d x, d s) \\
\tilde{X}_{t}^{\varepsilon} & =\int_{0}^{t} \int_{\varepsilon \leq|x|<1} x\left\{J_{X}(d x, d s)-\nu_{X}(d x) d s\right\} .
\end{aligned}
$$

Furthermore, if the jump component has finite variation, i.e.

$$
\int_{|x|<1}|x| \nu_{X}(d x)<\infty
$$

then (1) can be rewritten as

$$
X_{t}=\left[\mu-\int_{|x|<1} x \nu(d x)\right] t+\sigma B_{t}+\int_{0}^{t} \int_{\mathbb{R}} x J_{X}(d x, d s) .
$$

Let $S_{t}=S_{0} \exp \left(X_{t}\right)$, Itô formula leads to the following stochastic differential equation

$$
\begin{aligned}
\frac{d S_{t}}{S_{t-}} & =\left[\mu+\frac{1}{2} \sigma^{2}+\int_{\mathbb{R}}\left(e^{x}-1-x \cdot 1_{|x|<1}\right) \nu_{X}(d x)\right] d t+\sigma d B_{t}+\int_{\mathbb{R}}\left(e^{x}-1\right)\left[J_{X}(d x, d t)-\nu_{X}(d x) d t\right] \\
& =(r+\phi) d t+\sigma d B_{t}+\int_{\mathbb{R}}\left(e^{x}-1\right)\left[J_{X}(d x, d t)-\nu_{X}(d x) d t\right]
\end{aligned}
$$

where we let

$$
\phi=\mu+\frac{1}{2} \sigma^{2}+\int_{\mathbb{R}}\left(e^{x}-1-x \cdot 1_{|x|<1}\right) \nu_{X}(d x)-r
$$

denote the equity premium. Additionally, there is a money market account $\left\{M_{t}\right\}_{t \geq 0}$ which allows both borrowing and lending at a risk-free rate $r$, and it follows

$$
\frac{d M_{t}}{M_{t}}=r d t
$$

\section{Equity Premium and Pricing Kernel}

\subsection{Equilibrium Approach of Asset Pricing}

To apply the equilibrium approach, we consider a representative investor trading in both of the risky asset and the risk-free money account and consuming over the time horizon $[0, T]$ as well. Let $\left\{c_{t}\right\}_{t \geq 0}$ be the consumption 
rate, and $\left\{W_{t}\right\}_{t \geq 0}$ be the wealth process of the representative investor with $W_{t}$ written as

$$
W_{t}=W_{1 t}+W_{2 t}
$$

where $W_{1 t}=\omega W_{t}$ and $W_{2 t}=(1-\omega) W_{t}$ denote the wealth invested in the risky stock and risk-free money account respectively. Hence, (3) and (5) lead to

$$
\frac{d W_{t}}{W_{t-}}=\left[r+\omega \phi-\omega \int_{\mathbb{R}}\left(e^{x}-1\right) \nu_{X}(d x)-\frac{c_{t}}{W_{t-}}\right] d t+\omega \sigma d B_{t}+\omega \int_{\mathbb{R}}\left(e^{x}-1\right) J_{X}(d x, d t),
$$

where both of the percentage of wealth invested in the stock $\omega$ and the consumption rate $c_{t}$ are control variables.

Subject to (6), the object of the investor is to maximize the expected utility function of his consumption

$$
\max _{\left(\omega, c_{t}\right)} \mathbb{E}_{t}\left[\int_{t}^{T} p(s) U\left(c_{s}\right) d s\right]
$$

where $p(t)$ denotes the time preference function with $p(t) \geq 0$ on $[0, T]$, and $U(\cdot)$ is a utility function with $U^{\prime}>0$ and $U^{\prime \prime}>0$. Here we consider the constant relative risk aversion (CRRA) utility function

$$
U(c)= \begin{cases}\frac{c^{1-\gamma}}{1-\gamma}, & \gamma>0, \gamma \neq 1 \\ \ln c, & \gamma=1\end{cases}
$$

where $\gamma$ is called the relative risk aversion coefficient.

\subsection{Equity Premium}

By solving the problem of (7) and applying the market clearing condition $\omega=1$, we can obtain the equilibrium equity premium $\phi$, and furthermore analyze the effects of the diffusive risk and jump risk on this $\phi$.

Proposition 1. In the economy where there is a single production process given by (3) and the representative investor has CRRA utility function, the equilibrium equity premium is given by

$$
\phi=\gamma \sigma^{2}+\int_{\mathbb{R}}\left[\left(e^{x}-1\right)\left(1-e^{-\gamma x}\right)\right] \nu_{X}(d x),
$$

which is a sum of a diffusion risk premium and a rare-event risk premium.

Proof. Define

$$
V\left(t, W_{t}\right):=\max _{\left(\omega, c_{t}\right)} \mathbb{E}_{t}\left[\int_{t}^{T} p(s) U\left(c_{s}\right) d s\right]
$$

then it satisfies the following HJB equation

$$
\max _{\left(\omega, c_{t}\right)} \mathbb{E}_{t}\left[d V\left(t, W_{t}\right)+p(t) U\left(c_{t}\right) d t\right]=0
$$


where $d V\left(t, W_{t}\right)$ can be obtained as follows by applying Itô formula

$$
\begin{aligned}
d V\left(t, W_{t}\right)= & V_{t}\left(t, W_{t-}\right) d t+V_{W}\left(t, W_{t-}\right)\left\{\left[r+\omega \phi-\omega \int_{\mathbb{R}}\left(e^{x}-1\right) \nu_{X}(d x)-\frac{c_{t}}{W_{t-}}\right] W_{t-} d t+\omega \sigma W_{t-} d B_{t}\right\} \\
& +\frac{1}{2} V_{W W}\left(t, W_{t-}\right) \omega^{2} \sigma^{2} W_{t-}^{2} d t+\int_{\mathbb{R}}\left[V\left(t, W_{t-}\left(1+\omega\left(e^{x}-1\right)\right)\right)-V\left(t, W_{t-}\right)\right] J_{X}(d x, d t),
\end{aligned}
$$

where the subscripts are used to denote the partial derivatives. By considering the expectation on the left hand side of (10), we have the following first-order condition for this maximization problem

$$
\left\{\begin{array}{l}
-V_{W}+p(t) U^{\prime}\left(c_{t}\right)=0, \\
{\left[\phi-\int_{\mathbb{R}}\left(e^{x}-1\right) \nu_{X}(d x)\right] W V_{W}+\omega \sigma^{2} W^{2} V_{W W}+\int_{\mathbb{R}}\left[V_{W}\left(t, W\left(1+\omega\left(e^{x}-1\right)\right)\right) \cdot W \cdot\left(e^{x}-1\right)\right] \nu_{X}(d x)=0,}
\end{array}\right.
$$

where we have suppressed the dependence of $V\left(t, W_{t-}\right)$ on $t$ and $W_{t-}$, and the market clearing condition $\omega=1$ implies

$$
\phi=-\frac{1}{V_{W}}\left\{\sigma^{2} W V_{W W}+\int_{\mathbb{R}}\left[V_{W}\left(t, W e^{x}\right) \cdot\left(e^{x}-1\right)\right] \nu_{X}(d x)\right\}+\int_{\mathbb{R}}\left(e^{x}-1\right) \nu_{X}(d x) .
$$

The substitution of (12) and $\omega=1$ into (10) results in

$$
\begin{aligned}
0= & V_{t}+r W V_{W}-\frac{1}{2} \sigma^{2} W^{2} V_{W W}-W \int_{\mathbb{R}}\left[V_{W}\left(t, W e^{x}\right) \cdot\left(e^{x}-1\right)\right] \nu_{X}(d x) \\
& +\int_{\mathbb{R}}\left[V\left(t, W e^{x}\right)-V(t, W)\right] \nu_{X}(d x)-c_{t} V_{W}+p(t) U\left(c_{t}\right),
\end{aligned}
$$

and, by considering the two cases of $\gamma>0, \gamma \neq 1$ and $\gamma=1$ separately and appropriate conjecture, we can solve this partial integro-differential equation (PIDE) and finally obtain (8) by substituting the solution of this PIDE into (12).

For further analysis, we introduce the following notations for the diffusion risk premium and jump risk premium

$$
\phi_{D}=\gamma \sigma^{2}, \quad \phi_{J}=\int_{\mathbb{R}}\left[\left(e^{x}-1\right)\left(1-e^{-\gamma x}\right)\right] \nu_{X}(d x) .
$$

Remark 2. In constrast to Zhang et al. (2010) which uses a compound Poisson process, the jump risk premium $\phi_{J}$ given in the above allows an arbitrary distribution even with infinite activity for the jump intensity.

Remark 3. Since $\gamma>0$, the integrand $\left(e^{x}-1\right)\left(1-e^{-\gamma x}\right)$ in the $\phi_{J}$ is always positive for any value of $x$, which can be either positive or negative. And it means that both of a positive and a negative jump can result in a positive part of the risk premium. Furthermore, for any $x,\left(e^{x}-1\right)\left(1-e^{-\gamma x}\right)$ is an increasing function of $\gamma$, which is consistent with the fact that the more risk-averse is the investor, the higher is the risk premium.

Remark 4 . We can rewrite the jump risk premium $\phi_{J}$ as follows by using Taylor's formula

$$
\phi_{J}=\int_{\mathbb{R}}\left[\left(e^{x}-1\right)\left(1-e^{-\gamma x}\right)\right] \nu_{X}(d x)=\gamma \int_{\mathbb{R}} x^{2} \nu_{X}(d x)+\mathcal{O}\left(x^{4}\right),
$$

therefore when the jump size $x$ is small enough, the total risk premium can be approximated as follows

$$
\phi \approx \gamma\left(\sigma^{2}+\int_{\mathbb{R}} x^{2} \nu_{X}(d x)\right)=\gamma \frac{\operatorname{Var}\left(\ln \left(S_{t} / S_{0}\right)\right)}{t},
$$

which shows that the total risk premium, given by the annual variance of the log return multiplied by the risk aversion coefficient, does not tell the difference between the risk premium contributed by the diffusion risk and 
the one due to the jump risk with small jump sizes. This is nearly the same as mentioned in the corollary 3.7 in Zhang et al. (2010).

\subsection{Pricing Kernel}

Proposition 5. In the jump diffusion economy given by (3) and if the representative investor has CRRA utility function, the pricing kernel $\left\{\pi_{t}\right\}_{t \geq 0}$ is given by the following stochastic differential eqation

$$
\frac{d \pi_{t}}{\pi_{t-}}=\left[-r-\int_{\mathbb{R}}\left(e^{z}-1\right) \nu_{Z}(d z)\right] d t-\gamma \sigma d B_{t}+\int_{\mathbb{R}}\left(e^{z}-1\right) J_{Z}(d z, d t),
$$

where the jump component of $\pi$ is modeled by another jump process $Z$ with jump measure $J_{Z}$ and Lévy kernel $\nu_{Z}$, rather than the jump component of $X$, but the $B$ is the same Brownian motion as in $X$. To make $\pi S$ a martingale, i.e. $\pi_{t} S_{t}=\mathbb{E}_{t}\left[\pi_{T} S_{T}\right], \pi$ also needs to satisfy the following condition

$$
\int_{\mathbb{R}}\left(e^{x}-1\right) e^{-\gamma x} \nu_{X}(d x)=\int_{\mathbb{R}^{2}} e^{z}\left(e^{x}-1\right) \nu_{X, Z}(d x \times d z) .
$$

Proof. First, it is obvious that the above $\pi$ can lead to $\pi_{t} M_{t}=\mathbb{E}_{t}\left(\pi_{T} M_{T}\right)$. Second, the dynamics of $\pi S$ can be obtained as

$$
\begin{aligned}
\frac{d\left(\pi_{t} S_{t}\right)}{\pi_{t-} S_{t-}}= & {\left[-\int_{\mathbb{R}}\left(e^{z}-1\right) \nu_{Z}(d z)-\int_{\mathbb{R}}\left(e^{x}-1\right) e^{-\gamma x} \nu_{X}(d x)\right] d t+\gamma \sigma d B_{t} } \\
& +\int_{\mathbb{R}}\left(e^{z}-1\right) J_{Z}(d z, d t)+\int_{\mathbb{R}}\left(e^{x}-1\right) J_{X}(d x, d t)+\int_{\mathbb{R}^{2}}\left(e^{x}-1\right)\left(e^{z}-1\right) J_{X, Z}(d x \times d z, d t)
\end{aligned}
$$

and to make is a martingale, we need

$$
\int_{\mathbb{R}}\left(e^{x}-1\right) e^{-\gamma x} \nu_{X}(d x)=\int_{\mathbb{R}^{2}} e^{z}\left(e^{x}-1\right) \nu_{X, Z}(d x \times d z) .
$$

Remark 6. In Pan (2002), Liu and Pan (2003) and Liu et al. (2005), strict assumptions are imposed on the distribution of the jump size for deriving the pricing kernel under jump diffusion model, and although Zhang et al. (2010) removes these restrictions, it only uses a compound Poisson distribution for the jump component. Therefore, this result is comparatively general.

Remark 7. Obviously, the distribution of the jump process $Z$, satisfying the conditon of (15), is not unique, and this is due to the the presence of the jump component in $X$ which makes the market incomplete.

\section{Option Pricing}

In this section, we will first provide the equilibrium option pricing formula by using the pricing kernel derived above, and then show that this equilibrium result is actually equivalent to the risk-neutral result obtained by Bakshi and Madan (2000). 


\subsection{Equilibrium Approach}

Let $C(S, t)$ denote the time- $t$ value of a European call option with spot price $S$, then based on that $C(S, t) \pi_{t}$ is a martingale, we have the following proposition which contains a PIDE and an explicit formula for the option price.

Since in the subsequent sections we have to use the Lévy kernel under the risk-neutral probability $\mathbb{Q}$, before introducing the following proposition we first define the risk-neutral probability $\mathbb{Q}$ as

$$
\left.\frac{d \mathbb{Q}}{d \mathbb{P}}\right|_{\mathcal{F}_{t}}=e^{r t} \pi_{t}=\exp \left\{\left[-\frac{1}{2} \gamma^{2} \sigma^{2}-\int_{\mathbb{R}}\left(e^{z}-1\right) \nu_{Z}(d z)\right] t-\gamma \sigma B_{t}+\int_{0}^{t} \int_{\mathbb{R}} z J_{Z}(d z, d s)\right\},
$$

where the pricing kernel or the distribution of $Z$ is not unique in this incomplete market, and thus the riskneutral measure $\mathbb{Q}$ is also not unique. Under the measure $\mathbb{Q},\left\{\widetilde{B}_{t}=B_{t}+\gamma \sigma t\right\}_{t \geq 0}$ is a standard Brownian motion, and the distribution of the jump component in $X$ can be derived as

$$
\mathbb{E}^{\mathbb{Q}}\left\{\exp \left[\theta \int_{0}^{t} \int_{\mathbb{R}} x J_{X}(d x, d s)\right]\right\}=\exp \left[\int_{\mathbb{R}^{2}} e^{z}\left(e^{\theta x}-1\right) \nu_{X, Z}(d x \times d z) \cdot t\right],
$$

therefore it follows that

$$
\nu_{X}^{\mathbb{Q}}(d x)=\int_{\mathbb{R}} e^{z} \nu_{X, Z}(d x \times d z),
$$

and based on (15) we have

$$
\int_{\mathbb{R}}\left(e^{x}-1\right) e^{-\gamma x} \nu_{X}(d x)=\int_{\mathbb{R}}\left(e^{x}-1\right) \nu_{X}^{\mathbb{Q}}(d x) .
$$

Proposition 8. In the above jump diffusion economy, the price of a European call option satisfies the following PIDE

$$
C_{t}+\left[r-\int_{\mathbb{R}}\left(e^{x}-1\right) \nu_{X}^{\mathbb{Q}}(d x)\right] S C_{s}+\frac{1}{2} \sigma^{2} S^{2} C_{s s}-r C+\int_{\mathbb{R}}\left[C\left(S e^{x}, t\right)-C(S, t)\right] \nu_{X}^{\mathbb{Q}}(d x)=0
$$

with a terminal condition

$$
C(S, T)=\max (S-K, 0)
$$

where $\nu_{X}^{\mathbb{Q}}$ is the Lévy kernel under the risk-neutral probability $\mathbb{Q}$ defined by (16) and (17).

And an explicit result, called as modified Merton (1976)'s formula for the call option price, can be obtained as

$$
C(S, t)=\mathbb{E}\left\{\exp \left[\widetilde{Z}_{\tau}\right] \cdot C^{B S}\left(S \cdot \exp \left(\tilde{X}_{\tau}\right), t\right)\right\}
$$

where

$$
\begin{aligned}
\tau & =T-t \\
\widetilde{X}_{\tau} & =\int_{0}^{\tau} \int_{\mathbb{R}} x J_{X}(d x, d s)-\tau \int_{\mathbb{R}}\left(e^{x}-1\right) \nu_{X}^{\mathbb{Q}}(d x), \\
\widetilde{Z}_{\tau} & =\int_{0}^{\tau} \int_{\mathbb{R}} z J_{Z}(d z, d s)-\tau \int_{\mathbb{R}}\left(e^{z}-1\right) \nu_{Z}(d z),
\end{aligned}
$$

and $C^{B S}(\cdot, \cdot)$ is the Black-Scholes formula for the European call option.

Proof. Firstly, for the PIDE (19), the dynamics of $C\left(S_{t}, t\right) \pi_{t}$ can be derived as 


$$
\begin{aligned}
d\left(C\left(S_{t}, t\right) \pi_{t}\right)= & C\left(S_{t-}, t\right) d \pi_{t}^{c}+\pi_{t-} d C^{c}\left(S_{t}, t\right)+\left(d \pi_{t}^{c} \cdot d C^{c}\left(S_{t}, t\right)\right)+\triangle\left(C\left(S_{t}, t\right) \pi_{t}\right) \\
= & C\left(S_{t-}, t\right) \pi_{t-}\left[\left(-r-\int_{\mathbb{R}}\left(e^{z}-1\right) \nu_{Z}(d z)\right) d t-\gamma \sigma d B_{t}\right] \\
& +\pi_{t-} C_{s}\left(S_{t-}, t\right) S_{t-}\left[(r+\phi) d t+\sigma d B_{t}-\int_{\mathbb{R}}\left(e^{x}-1\right) \nu_{X}(d x) d t\right] \\
& +\pi_{t-}\left[C_{t}\left(S_{t-}, t\right) d t+\frac{1}{2} C_{s s}\left(S_{t-}, t\right) S_{t-}^{2} \sigma^{2} d t\right]-\gamma \sigma^{2} \pi_{t-} C_{s}\left(S_{t-}, t\right) S_{t-} d t \\
& +\pi_{t-} \int_{\mathbb{R}^{2}}\left[e^{z} C\left(S_{t-} e^{x}, t\right)-C\left(S_{t-}, t\right)\right] J_{X, Z}(d x \times d z, d t),
\end{aligned}
$$

where the superscript $c$ stands for the corresponding process without jump, and $\triangle\left(C\left(S_{t}, t\right) \pi_{t}\right)$ represents the jump component of the dynamics of $C\left(S_{t}, t\right) \pi_{t}$ at time $t$. Since it is a martingale, by substituting (8) we have

$$
C_{t}+\left[r-\int_{\mathbb{R}}\left(e^{x}-1\right) e^{-\gamma x} \nu_{X}(d x)\right] S C_{s}+\frac{1}{2} \sigma^{2} S^{2} C_{s s}-r C+\int_{\mathbb{R}^{2}} e^{z}\left[C\left(S e^{x}, t\right)-C(S, t)\right] \nu_{X, Z}(d x \times d z)=0
$$

and the PIDE (19) follows from the condition (17) and (18).

Secondly, for the explicit result (20), according to (14) and (3) we have

$$
\begin{aligned}
\frac{\pi_{T}}{\pi_{t}} & =\exp \left\{\left[-r-\frac{1}{2} \gamma^{2} \sigma^{2}-\int_{\mathbb{R}}\left(e^{z}-1\right) \nu_{Z}(d z)\right] \tau-\gamma \sigma\left(B_{T}-B_{t}\right)+\int_{t}^{T} \int_{\mathbb{R}} z J_{Z}(d z, d s)\right\}, \\
\frac{S_{T}}{S_{t}} & =\exp \left\{\left[r+\phi-\frac{1}{2} \sigma^{2}-\int_{\mathbb{R}}\left(e^{x}-1\right) \nu_{X}(d x)\right] \tau+\sigma\left(B_{T}-B_{t}\right)+\int_{t}^{T} \int_{\mathbb{R}} x J_{X}(d x, d s)\right\} \\
& =\exp \left\{\left[r-\frac{1}{2} \sigma^{2}-\int_{\mathbb{R}}\left(e^{x}-1\right) \nu_{X}^{\mathbb{Q}}(d x)\right] \tau+\sigma\left(\widetilde{B}_{T}-\widetilde{B}_{t}\right)+\int_{t}^{T} \int_{\mathbb{R}} x J_{X}(d x, d s)\right\},
\end{aligned}
$$

where

$$
\widetilde{B}_{t}=B_{t}+\gamma \sigma t
$$

and then we can calculate $\mathbb{E}_{t}\left[\frac{\pi_{T}}{\pi_{t}}\left(S_{T}-K\right)^{+}\right]$as

$$
\begin{aligned}
C(S, t) & =\mathbb{E}_{t}\left[\frac{\pi_{T}}{\pi_{t}}\left(S_{T}-K\right)^{+}\right] \\
& =\exp \left\{\left[-\int_{\mathbb{R}}\left(e^{z}-1\right) \nu_{Z}(d z)\right] \tau\right\} \\
& \times \mathbb{E}_{t}\left\{\left.\exp \left[\int_{t}^{T} \int_{\mathbb{R}} z J_{Z}(d z, d s)\right] \cdot \frac{d \mathbb{H}}{d \mathbb{P}}\right|_{\mathcal{F}_{t}} \cdot e^{-r \tau}\left[S_{T}^{B S} \cdot \exp \left(\int_{t}^{T} \int_{\mathbb{R}} x J_{X}(d x, d s)-\tau \int_{\mathbb{R}}\left(e^{x}-1\right) \nu_{X}^{\mathbb{Q}}(d x)\right)-K\right]^{+}\right\} \\
& =\mathbb{E}\left\{\exp \left[\widetilde{Z}_{\tau}\right] \cdot C^{B S}\left(S \cdot \exp \left(\widetilde{X}_{\tau}\right), t\right)\right\},
\end{aligned}
$$

where

$$
\begin{aligned}
\left.\frac{d \mathbb{H}}{d \mathbb{P}}\right|_{\mathcal{F}_{t}} & =\exp \left\{-\frac{1}{2} \gamma^{2} \sigma^{2} \tau-\gamma \sigma\left(B_{T}-B_{t}\right)\right\} \\
S_{T}^{B S} & =S_{t} \exp \left\{\left(r-\frac{1}{2} \sigma^{2}\right) \tau+\sigma\left(\widetilde{B}_{T}-\widetilde{B}_{t}\right)\right\}
\end{aligned}
$$


and under the measure $\mathbb{H},\left\{\widetilde{B}_{t}\right\}$ is a standard Brownian motion whereas the distributions of the jump components in $X$ and $Z$ remain the same as in $\mathbb{P}$, and this allows the application of the Black-Scholes formula $C^{B S}(\cdot, \cdot)$.

\subsection{Risk-Neutral Approach}

By using the inverse Fourier transformation, Bakshi and Madan (2000) provides a general pricing formula for the European call option, which is represented in terms of the risk-neutral distribution of the log return. In our setting, where the underlying price is modeled as the exponential of a Lévy process, we can show that this risk-neutral approach is equivalent to the above equilibrium approach with its result given by (20).

Proposition 9. The call option price given by (20) is equivalent to the Bakshi and Madan (2000)'s result, which shows that the price of a European call option can be given by

$$
C(S, t)=S \Pi_{1}-K e^{-r \tau} \Pi_{2},
$$

where

$$
\begin{aligned}
\Pi_{1} & =\frac{1}{2}+\frac{1}{\pi} \int_{0}^{+\infty} \operatorname{Re}\left[\frac{e^{-i \theta \ln (K / S)} \times f^{\mathbb{Q}}(\theta-i, \tau)}{i \theta f^{\mathbb{Q}}(-i, \tau)}\right] d \theta, \\
\Pi_{2} & =\frac{1}{2}+\frac{1}{\pi} \int_{0}^{+\infty} \operatorname{Re}\left[\frac{e^{-i \theta \ln (K / S)} \times f^{\mathbb{Q}}(\theta, \tau)}{i \theta}\right] d \theta, \\
f^{\mathbb{Q}}(\theta, \tau) & =\mathbb{E}^{\mathbb{Q}}\left\{\exp \left[i \theta \ln \left(S_{T} / S_{t}\right)\right]\right\} .
\end{aligned}
$$

Proof. Note that

$$
\frac{S_{T}}{S_{t}}=\exp \left\{\left[r-\frac{1}{2} \sigma^{2}-\int_{\mathbb{R}}\left(e^{x}-1\right) \nu_{X}^{\mathbb{Q}}(d x)\right] \tau+\sigma\left(\widetilde{B}_{T}-\widetilde{B}_{t}\right)+\int_{t}^{T} \int_{\mathbb{R}} x J_{X}(d x, d s)\right\},
$$

we can show that

$$
f^{\mathbb{Q}}(\theta, \tau)=\mathbb{E}^{\mathbb{Q}}\left\{\exp \left[-\frac{1}{2} \sigma^{2} \theta^{2} \tau+i \theta\left(\widetilde{X}_{\tau}+r \tau-\frac{1}{2} \sigma^{2} \tau\right)\right]\right\} .
$$

Then by substitution, we can calculate $\Pi_{2}$ as follows

$$
\begin{aligned}
\Pi_{2} & =\frac{1}{2}+\frac{1}{\pi} \int_{0}^{+\infty} \operatorname{Re}\left[\frac{e^{-i \theta \ln (K / S)} \times f^{\mathbb{Q}}(\theta, \tau)}{i \theta}\right] d \theta \\
& =\frac{1}{2}+\frac{1}{\pi} \int_{0}^{+\infty} \mathbb{E}^{\mathbb{Q}}\left\{\operatorname{Re}\left\{\frac{1}{i \theta} \exp \left[-\frac{1}{2} \sigma^{2} \theta^{2} \tau+i \theta\left(\ln (S / K)+\widetilde{X}_{\tau}+r \tau-\frac{1}{2} \sigma^{2} \tau\right)\right]\right\}\right\} d \theta \\
& =\frac{1}{2}+\frac{1}{\pi} \int_{0}^{+\infty} \mathbb{E}\left\{\operatorname{Re}\left[\frac{1}{i \theta} \exp \left(-\frac{1}{2} \sigma^{2} \theta^{2} \tau+\widetilde{Z}_{\tau}+i \theta u_{2}\right)\right]\right\} d \theta \\
& =\frac{1}{2}+\frac{1}{\pi} \int_{0}^{+\infty} \mathbb{E}\left[\exp \left(-\frac{1}{2} \sigma^{2} \theta^{2} \tau+\widetilde{Z}_{\tau}\right) \frac{\sin \left(\theta u_{2}\right)}{\theta}\right] d \theta \\
& =\frac{1}{2}+\mathbb{E}\left[\exp \left(\widetilde{Z}_{\tau}\right) \times\left(N\left(d_{2}\right)-\frac{1}{2}\right)\right] \\
& =\mathbb{E}\left[\exp \left(\widetilde{Z}_{\tau}\right) \times N\left(d_{2}\right)\right],
\end{aligned}
$$


where $N(\cdot)$ is the cumulative distribution function of the standard normal distribution and

$$
\begin{aligned}
u_{2} & =\ln (S / K)+\widetilde{X}_{\tau}+r \tau-\frac{1}{2} \sigma^{2} \tau \\
& =\ln \left(\frac{S \cdot \exp \left(\widetilde{X}_{\tau}\right)}{K \cdot \exp (-r \tau)}\right)-\frac{1}{2} \sigma^{2} \tau, \\
d_{2} & =\frac{u_{2}}{\sigma \sqrt{\tau}},
\end{aligned}
$$

and similarly for the calculation of $\Pi_{1}$, then the equivalence between (20) and (24) follows.

\section{Explanation of The Empirical Evidence}

To explain the empirical evidence such as the negative variance risk premium, implied volatility smirk, and negative skewness risk premium based on the above derived risk-neutral distribution, we first provide the relations between the physical and risk-neutral mean, variance and skewness of the stock return in the following proposition, where we use $X_{t}$ to denote the continuously compounded return within $(0, t)$ since $X_{t}=\ln S_{t} / S_{0}$.

Proposition 10. The mean, variance, and skewness of $X_{t}$ under the physical probability are given by

$$
\begin{aligned}
\mathbb{E}\left[X_{t}\right] & =\left[r+\phi-\frac{1}{2} \sigma^{2}-\int_{\mathbb{R}}\left(e^{x}-1-x\right) \nu_{X}(d x)\right] t, \\
\operatorname{Var}\left[X_{t}\right] & =\left[\sigma^{2}+\int_{\mathbb{R}} x^{2} \nu_{X}(d x)\right] t, \\
\text { Skewness }\left[X_{t}\right] & =\frac{\left[\int_{\mathbb{R}} x^{3} \nu_{X}(d x)\right] t^{-\frac{1}{2}}}{\left[\sigma^{2}+\int_{\mathbb{R}} x^{2} \nu_{X}(d x)\right]^{\frac{3}{2}}},
\end{aligned}
$$

and under the risk-neutral measure (16), they can be given by

$$
\begin{aligned}
\mathbb{E}^{\mathbb{Q}}\left[X_{t}\right] & =\left[r-\frac{1}{2} \sigma^{2}-\int_{\mathbb{R}}\left(e^{x}-1-x\right) \nu_{X}^{\mathbb{Q}}(d x)\right] t, \\
\operatorname{Var}^{\mathbb{Q}}\left[X_{t}\right] & =\left[\sigma^{2}+\int_{\mathbb{R}} x^{2} \nu_{X}^{\mathbb{Q}}(d x)\right] t, \\
\text { Skewness }^{\mathbb{Q}}\left[X_{t}\right] & =\frac{\left[\int_{\mathbb{R}} x^{3} \nu_{X}^{\mathbb{Q}}(d x)\right] t^{-\frac{1}{2}}}{\left[\sigma^{2}+\int_{\mathbb{R}} x^{2} \nu_{X}^{\mathbb{Q}}(d x)\right]^{\frac{3}{2}}} .
\end{aligned}
$$

Proof. Based on the expressions for $S$ of (22) and (23) under the physical measure and the risk-neutral measure respectively, the results follow immediately.

These results allow a general distribution for the jump component of $X$, and thus can be easily deduced to the simple cases such as that the jump component follows a compound Poission process, or even that the jump size is a constant. Now, by using these analytical results, we can explain the empirical evidence mentioned before. And whether the empirical evidence is consistent with the analytical results is essentially dependent on the comparison between the physical Lévy kernel $\nu_{X}$ and the risk-neutral Lévy kernel $\nu_{X}^{\mathbb{Q}}$. 
Therefore, for any physical distribution of the jump component of $X$, we can first obtain its risk-neutral distribution based on (18), and then use the results given in the above proposition 10 to explain the empirical evidence. In the following, we will first discuss a very simple case with constant jump size, and then a more general case with the jump component following a CGMY model.

\subsection{A Simple Case}

In the simplist case where the jump size is a constant $x$ and the jump density is modeled as the increment of a Poisson process with parameter $\lambda$, the condition of (18) can be deduced to

$$
\lambda^{\mathbb{Q}}=e^{-\gamma x} \lambda\left\{\begin{array}{ll}
>\lambda & x<0 \\
<\lambda & x>0
\end{array},\right.
$$

where $\lambda^{\mathbb{Q}}$ is the risk-neutral parameter of the Poisson process. An intuitive explanation is that if the jump size is negative (positive), which is regarded as a bad (good) event, then it occurs more (less) frequently in the risk-neutral world than in the physical world due to the presence of the risk aversion. And for a fixed value of $x$, the larger is $\gamma$, the larger is the difference between the physical and risk-neutral probabilities.

Proposition 11. For the jump component of $X$ which follows a Poisson distribution with constant jump size $x$, the variance risk premium for the time horizon $[0, t]$ is given by

$$
x^{2}\left(1-e^{-\gamma x}\right) \lambda t
$$

and thus we have the negative variance risk premium (the risk-neutral variance is larger then the physical variance) if and only if

$$
x<0 \text {. }
$$

And the risk-neutral skewness is negative (implied volatility smirk) if and only if

$$
x<0 \text {. }
$$

Proof. The conclusions follows easily from the proposition 10.

This result is consistent with the one given in Zhang et al. (2010), which says that an expectation of a market crash can explain the two phenomena observed in the market: the negative variance risk premium and the implied volatility smirk.

\subsection{CGMY Model}

A more general case can be considered if we use a CGMY model developed by Carr et al. (2002), and the Lévy kernel is given by

$$
\nu(x)=\left\{\begin{array}{ll}
C \frac{\exp (-G|x|)}{|x|^{1+Y}} & x<0 \\
C \frac{\exp (-M|x|)}{|x|^{1+Y}} & x>0
\end{array},\right.
$$




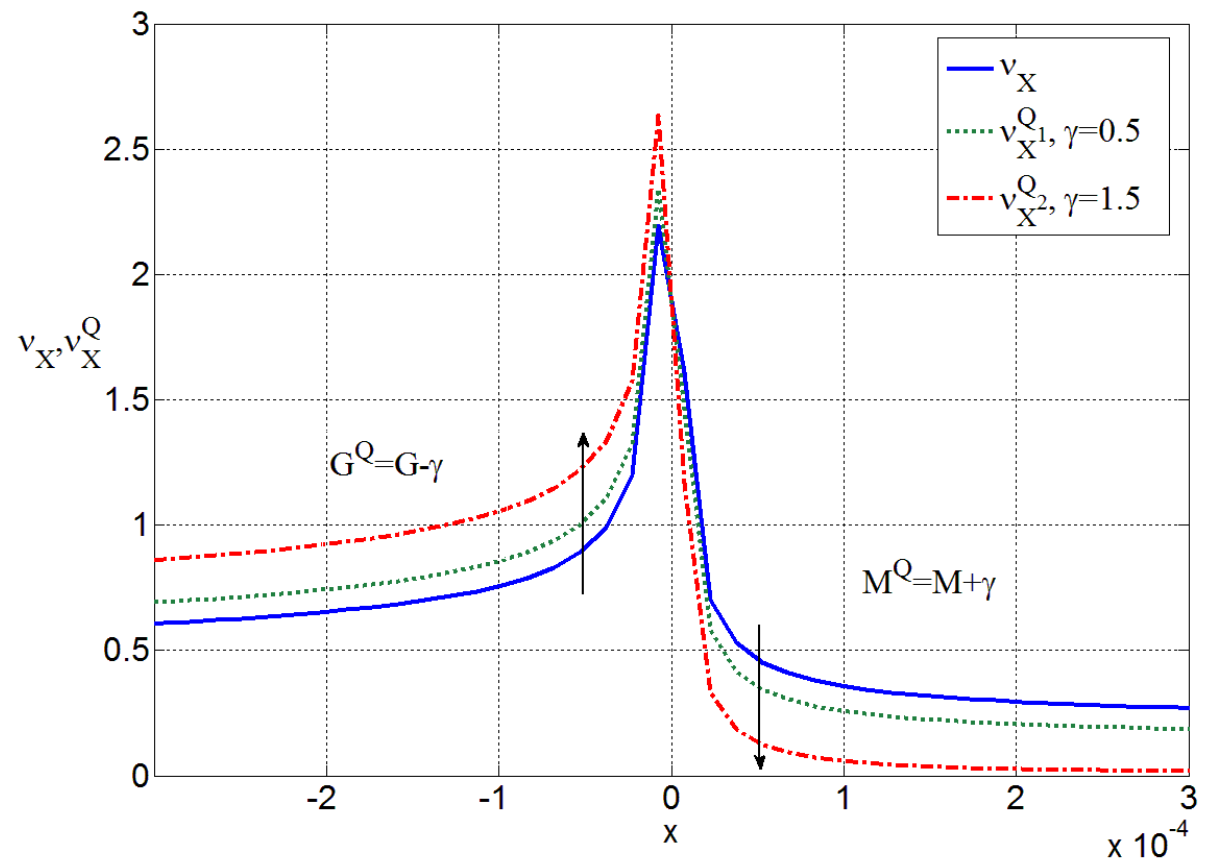

Figure 1: Comparison between the physical and the risk-neutral distributions for CGMY model

where $C>0, G>0, M>0$, and $Y<2$. Based on this, the variance and skewness of $X_{t}$ are given by

$$
\begin{aligned}
\operatorname{Var}\left[X_{t}\right] & =\left[\sigma^{2}+C \cdot \Gamma(2-Y) \cdot\left(\frac{1}{M^{2-Y}}+\frac{1}{G^{2-Y}}\right)\right] t, \\
\text { Skewness }\left[X_{t}\right] & =\frac{\left[C \cdot \Gamma(3-Y) \cdot\left(\frac{1}{M^{3-Y}}-\frac{1}{G^{3-Y}}\right)\right] t^{-\frac{1}{2}}}{\left[\sigma^{2}+C \cdot \Gamma(2-Y) \cdot\left(\frac{1}{M^{2-Y}}+\frac{1}{G^{2-Y}}\right)\right]^{\frac{3}{2}}} .
\end{aligned}
$$

Obviously, under the risk-neutral probability, the jump component will still follow the CGMY model but with different parameters. By using (18), we have the parameters under the risk-neutral measure given by

$$
\begin{aligned}
C^{\mathbb{Q}} & =C, \\
Y^{\mathbb{Q}} & =Y, \\
G^{\mathbb{Q}} & =G-\gamma, \\
M^{\mathbb{Q}} & =M+\gamma,
\end{aligned}
$$

where we need to impose an additional assumption of $G>\gamma$, and this result means that, due to the presence of the risk aversion, the negative (positive) jump has a smaller (larger) control rate of the exponential decay, which implies a fatter (thinner) tail and higher (lower) frequency of large jumps in the risk-neutral world than in the physical world. To illustrate this comparison, in the figure 1 for a CGMY model with $C=0.05, G=2$, $M=4$, and $Y=0.15$, we depict the original Lévy kernel under the physical measure and another two riskneutral kernels with respect to two values of the risk-aversion, and from which we can observe the effect of the risk aversion on the difference between the physical and the risk-neutral measures. From this difference, it follows the conditions equivalent to the negative variance risk premium and implied volatility smirk as given in proposition 13. 
Proposition 12. For the jump component of $X$ which follows a $C G M Y$ model with $C>0, G>\gamma, M>0$, and $Y<2$, the variance risk premium for the time horizon $[0, t]$ is given by

$$
C \cdot \Gamma(2-Y) \cdot\left[\left(\frac{1}{M^{2-Y}}-\frac{1}{(M+\gamma)^{2-Y}}\right)-\left(\frac{1}{(G-\gamma)^{2-Y}}-\frac{1}{G^{2-Y}}\right)\right] t,
$$

and thus we have the negative variance risk premium if and only if

$$
G<M+\gamma .
$$

And the risk-neutral skewness is negative (implied volatility smirk) if and only if

$$
G<M+2 \gamma
$$

Proof. First, from (27), the expression for the variance risk premium given in this proposition is obviously true. And the negative variance risk premium is equivalent to

$$
f(M+\gamma)<f(G)
$$

where

$$
f(x)=\frac{1}{(x-\gamma)^{2-Y}}-\frac{1}{x^{2-Y}}
$$

is a decreasing function for $x>\gamma$, therefore (29) follows. Second, from (28), we have the implied volatility smirk if and only if

$$
\frac{1}{\left(M^{\mathbb{Q}}\right)^{3-Y}}-\frac{1}{\left(G^{\mathbb{Q}}\right)^{3-Y}}<0,
$$

and this is obviously equivalent to (30).

Remark 13. For both of the simple case and the CGMY model, a careful analysis of the risk-neutral variance shows that given the negative variance risk premium (under the condition of $x<0$ for the simple case and $G<M+\gamma$ for the CGMY model), the risk-neutral variance increases with the value of the relative risk aversion coefficient $\gamma$. It means that the more risk-averse is the investor, the larger is the variance risk assumed in its risk-neutral world compared to the one in the physical world.

Remark 14. We have intuitive explanations for the above proposition. For the negative variance risk premium, in spite that the positive jump has a thinner tail in the risk-neutral world than in the physical world, the negative jump does the opposite and if it decays not too fast compared to the the positive jump ( $G$ is not too large comparted to $M$ ), then the total variance will still increase when we change from physical world to the risk-neutral world. And for the implied volatility smirk, the condition (30), equivalent to $G^{\mathbb{Q}}<M^{\mathbb{Q}}$, is consistent with the intuition that if, under the risk-neutral measure, the negative jump has smaller rate of decay and hence fatter tail than the positive jump, then the distribution is negatively skewed.

Different from these two empirical evidence, the situation for the negative skewness risk premium is a little bit complex. Due to the expressions of (25) and (26), the risk-neutral skewness is more negative than the physical one if and only if we have

$$
\frac{\int_{\mathbb{R}} x^{3} \nu_{X}^{\mathbb{Q}}(d x)}{\left[\sigma^{2}+\int_{\mathbb{R}} x^{2} \nu_{X}^{\mathbb{Q}}(d x)\right]^{\frac{3}{2}}}<\frac{\int_{\mathbb{R}} x^{3} \nu_{X}(d x)}{\left[\sigma^{2}+\int_{\mathbb{R}} x^{2} \nu_{X}(d x)\right]^{\frac{3}{2}}}<0,
$$


which is not only related to the relation between $\nu_{X}^{\mathbb{Q}}$ and $\nu_{X}$, but also related to the comparison between the jump risk and diffusion risk. The first two phenomena can be well explained by only using the distribution of the jump component, and the diffusion term has no contribution, but in constrast to them, we will not have the negative skewness risk premium by only specifying appropriate parameters for the jump component, since the diffusion risk is also involved.

\subsection{An Empirical Application}

To observe the above mentioned empirical evidence, we apply a jump diffusion process with the jump component following the CGMY model to the daily log returns of the S\&P 500 index from January 2, 1985 to December 30, 2011. By estimating the parameters, we can determine the coefficient of the risk aversion and make a comparison between the estimated physical and risk-neutral distributions.

By the inverse Fourier transformation, the probability density function of the daily $\log$ return $X_{\tau}=$ $\ln \left(S_{t+\tau} / S_{t}\right)$ with $\tau=\frac{1}{252}$ can be given by

$$
p(x)=\frac{1}{\pi} \int_{0}^{\infty} \operatorname{Re}[\exp (-i \theta x) \cdot f(\theta, \tau)] d \theta
$$

where $f(\theta, \tau)=\mathbb{E}\left\{\exp \left[i \theta X_{\tau}\right]\right\}$ is the characteristic function of $X_{\tau}$, and it can be shown that

$$
f(\theta, \tau)=\exp \left\{i \theta\left(r+\phi-\frac{1}{2} \sigma^{2}\right) \tau-\frac{1}{2} \sigma^{2} \theta^{2} \tau+\tau \int_{\mathbb{R}}\left[\left(e^{i \theta x}-1\right)-i \theta\left(e^{x}-1\right)\right] \nu_{X}(d x)\right\},
$$

where we take $r=0.0492$ which is the average 3-month yield, and for the integral we note that

$$
\int_{\mathbb{R}}\left(e^{i \theta x}-1\right) \nu_{X}(d x)=C \cdot \Gamma(-Y) \cdot\left[(M-i \theta)^{Y}-M^{Y}+(G+i \theta)^{Y}-G^{Y}\right],
$$

and $p(x)$ can be calculated by using fast Fourier transform.

Given the daily $\log$ returns $\left\{x_{n}\right\}_{n=1}^{N}$, we define the log likelihood function as

$$
L(\phi, \sigma, C, G, M, Y)=\sum_{n=1}^{N} \ln \left[p\left(x_{n}\right)\right] .
$$

By maximizing this function, we can obtain the maximum likelihood estimators (MLE) as:

\begin{tabular}{|c|c|c|c|c|c|}
\hline$\phi$ & $\sigma$ & $C$ & $G$ & $M$ & $Y$ \\
\hline 0.0438 & 0.1421 & 0.4672 & 24.4013 & 29.8832 & 0.2200 \\
\hline
\end{tabular}

and in the following figure 2, we depict the estimated density function with the observed density.

Based on these estimated parameters, we can obtain the risk aversion coefficient as follows:

$$
\phi=\gamma \sigma^{2}+\int_{\mathbb{R}}\left[\left(e^{x}-1\right)\left(1-e^{-\gamma x}\right)\right] \nu_{X}(d x) \Longrightarrow \gamma \approx \frac{\phi}{\sigma^{2}+C \cdot \Gamma(-Y) \cdot\left[\frac{1}{M^{2-Y}}+\frac{1}{G^{2-Y}}\right]}=1.9308
$$

and obviously we can have the conditions of (29) and (30) satisfied, which are consistent with the phenomena of the negative variance risk premium and implied volatility smirk. Furthermore, by using the previously derived relation between the physical and risk-neutral distribution, we can depict both of the physical and risk-neutral densities of the annual log return in the following figure 3. 


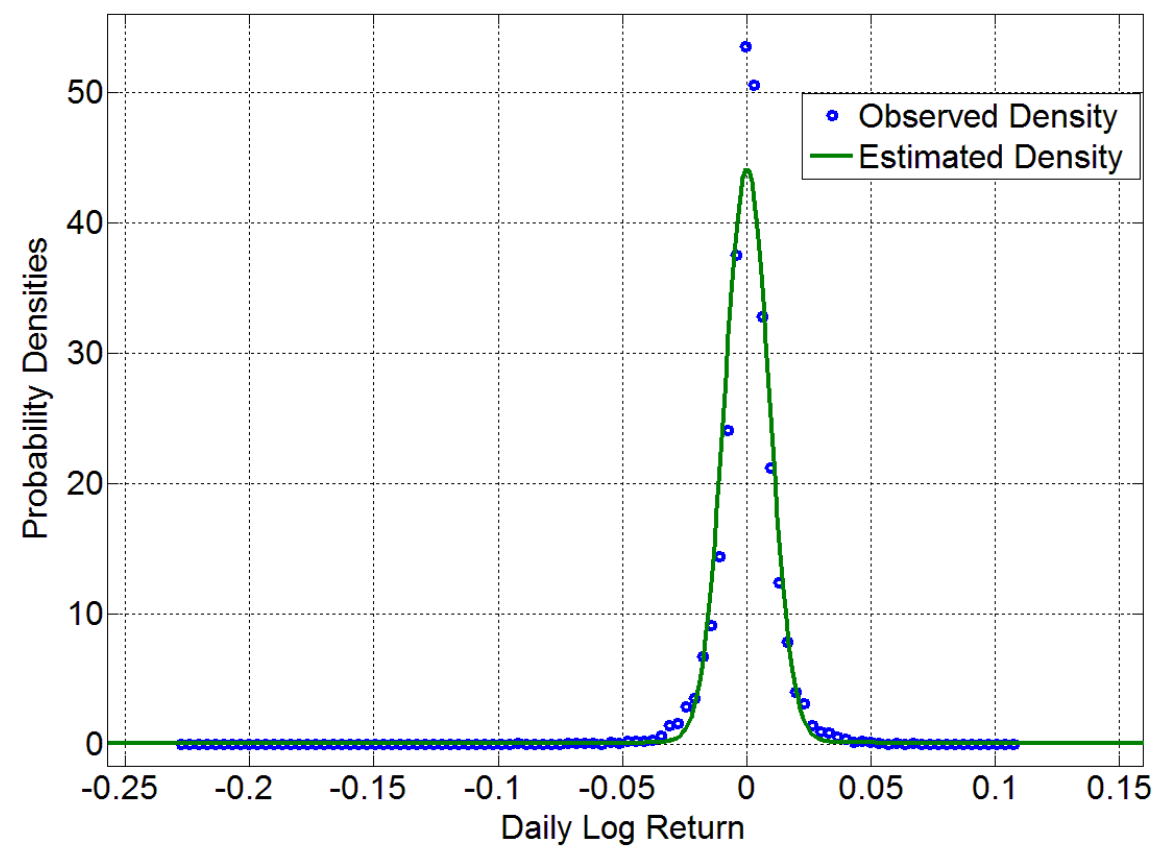

Figure 2: The density fit for a jump diffusion model (Brownian Motion and CGMY Model)

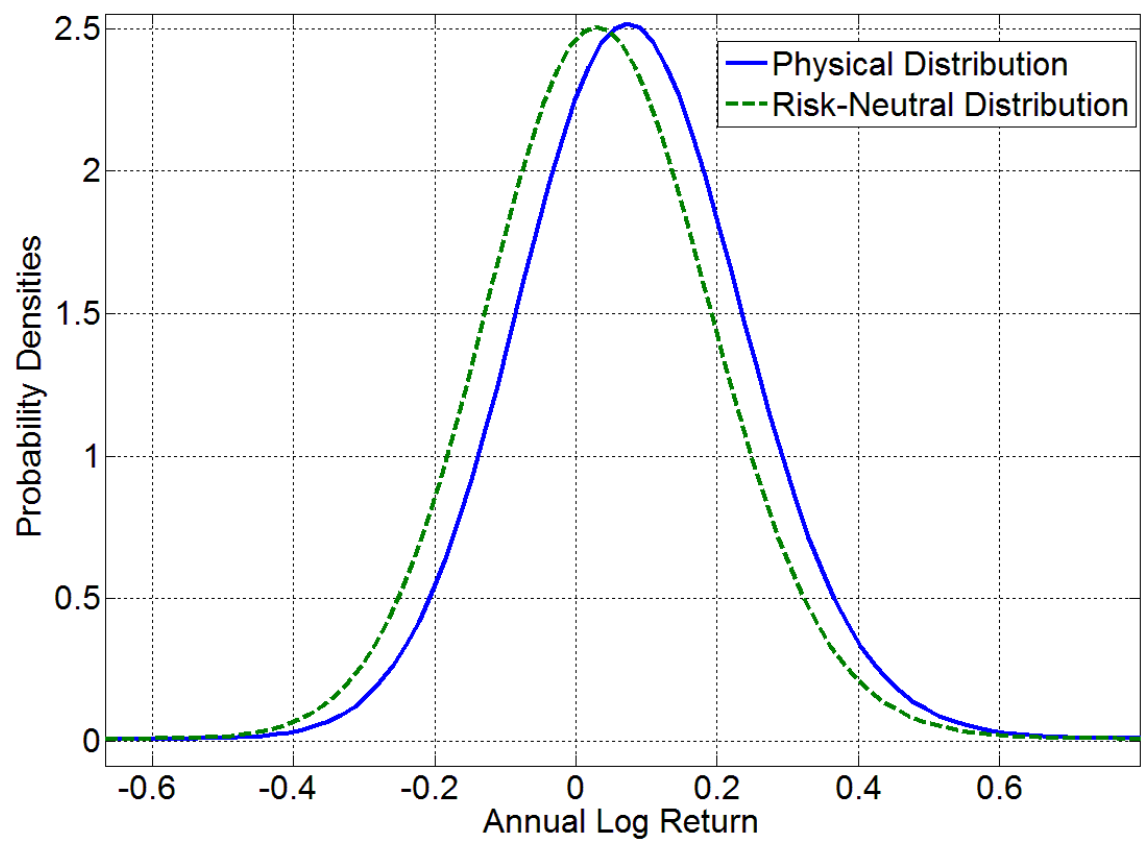

Figure 3: Comparison between the physical and the risk-neutral distributions of the annual log return 
Although, as mentioned in section 5.2, the condition for the negative skewness premium is more complex than the ones for the negative variance risk premium and the implied volatility smirk, by substituting the estimated parameters into (28), we can calculate the annual physical and risk-neutral skewnesses as

$$
\text { Skewness }\left[X_{1}\right]=-0.0166, \quad \text { Skewness }{ }^{\mathbb{Q}}\left[X_{1}\right]=-0.0309,
$$

which indicate a more negative risk-neutral skewness than the physical one, and this phenomenon can also be observed in the figure 3 .

\section{Conclusion}

As confirmed by previous studies, both of a continuous and a jump component exist in asset prices. And further observations support the evidence of negative variance risk premium, implied volatility smirk, and negative skewness risk premium. Therefore, this paper targets to explain these empirical phenomena under a comparatively general equilibrium model with both diffusion and jump risks.

In the production economy of Cox et al. (1985) where the single production process is modeled as the exponential of a Lévy process, we have successfully derived the equity premium and the pricing kernel with the jump component following an arbitrary distribution. By using this pricing kernel, an equilibrium option pricing formula can be obtained, and it can be regarded as a modified Merton (1976)'s result for the call option price by incorporating the risk-neutral distribution of the jump component. Moreover, this pricing formula can be shown to be equivalent to the Bakshi and Madan (2000)'s formula. In addition to that, the pricing kernel can also imply the transformation from the physical measure to the risk-neutral measure, and based on which, we can analyze the effect of the risk aversion on the difference between the two measures: the larger is the relative risk aversion coefficient, the higher (smaller) is the frequency of the negative (positive) jump in the risk neutral world than in the physical world. And this difference in the distribution of the jump component can perfectly explain the negative variance risk premium and implied volatility smirk, without any contribution from the diffusion risk. However, in contrast to this, the negative skewness risk premium is attributable to both of the jump and the diffusion risks.

Our model can be further extended to incorporate a stochastic volatility by using the time-changed Lévy process developed by Carr and $\mathrm{Wu}$ (2004), where the instantaneous rate of volatility is allowed to jump and even the volatility can be correlated with the log returns.

Acknowledgements: The authors would like to express deep gratitude to the two reviewers and the editor for their very helpful suggestions and comments. This research was supported by the Research Grants Council of the Hong Kong Special Administrative Region, China (project No. HKU 706611P).

\section{References}

Ait-Sahalia, Y. and Andrew, W. (1998). Nonparametric estimation of state-price densities implicit in financial asset prices. Journal of Finance, 53(2):499-547.

Altmann, T., Schmidt, T., and Stute, W. (2008). A shot noise model for financial assets. International Journal of Theoretical and Applied Finance, 11(1):87. 
Bakshi, G., Cao, C., and Chen, Z. (1997). Empirical performance of alternative option pricing models. Journal of finance, 52(5):2003-2049.

Bakshi, G. and Kapadia, N. (2003). Delta-hedged gains and the negative market volatility risk premium. Review of Financial Studies, 16(2):527-566.

Bakshi, G. and Madan, D. (2000). Spanning and derivative-security valuation. Journal of Financial Economics, 55(2):205-238.

Barndorff-Nielsen, O. (1997). Processes of normal inverse Gaussian type. Finance and stochastics, 2(1):41-68.

Carr, P., Geman, H., Madan, D., and Yor, M. (2002). The Fine Structure of Asset Returns: An Empirical Investigation. Journal of Business, 75(2):305-332.

Carr, P. and Wu, L. (2003). The finite moment log stable process and option pricing. Journal of Finance, 58(2):753-777.

Carr, P. and Wu, L. (2004). Time-changed Lévy processes and option pricing. Journal of Financial Economics, 71(1):113-142.

Carr, P. and Wu, L. (2009). Variance risk premiums. Review of Financial Studies, 22(3):13111341.

Coval, J. and Shumway, T. (2001). Expected option returns. The journal of Finance, 56(3):9831009.

Cox, J., Ingersoll Jr, J., and Ross, S. (1985). An intertemporal general equilibrium model of asset prices. Econometrica: Journal of the Econometric Society, 53(2):363-384.

Cox, J. and Ross, S. (1976). The valuation of options for alternative stochastic processes. Journal of financial Economics, 3(1-2):145-166.

Eberlein, E., Keller, U., and Prause, K. (1998). New insights into smile, mispricing, and value at risk: The hyperbolic model. The Journal of Business, 71(3):371-405.

Kou, S. and Wang, H. (2004). Option Pricing Under a Double Exponential Jump Diffusion Model. Management Science, 50(9):1178-1192.

Liu, J. and Pan, J. (2003). Dynamic derivative strategies. Journal of Financial Economics, 69(3):401-430.

Liu, J., Pan, J., and Wang, T. (2005). An equilibrium model of rare-event premia and its implication for option smirks. Review of Financial Studies, 18(1):131-164.

Madan, D., Carr, P., and Chang, E. (1998). The variance gamma process and option pricing. Review of Finance, 2(1):79. 
Martzoukos, S. and Trigeorgis, L. (2002). Real (investment) options with multiple sources of rare events. European Journal of Operational Research, 136(3):696-706.

Merton, R. (1976). Option pricing when underlying stock returns are discontinuous. Journal of Financial Economics, 3(1-2):125-144.

Morenno, M., Serrano, P., and Stute, W. (2011). Statistical properties and economic implications of jump-diffusion processes with shot-noise effects. European Journal of Operational Research, 214(3):656-664.

Pan, J. (2002). The jump-risk premia implicit in options: evidence from an integrated timeseries study. Journal of Financial Economics, 63(1):3-50.

Rossello, D. (2008). Maxvar with non-gaussian distributed returns. European Journal of Operational Research, 189(1):159-171.

Rubinstein, M. (1994). Implied binomial trees. Journal of finance, 49(3):771-818.

Zhang, J., Zhao, H., and Chang, E. (2010). Equilibrium asset and option pricing under jump diffusion. Mathematical Finance, 0(0):1-31. 